\title{
Detection and Monitoring of Repetitions Using an mHealth-Enabled Resistance Band
}

\author{
Curtis L. Petersen \\ The Dartmouth Institute \\ Quantitative Biomedical Science \\ Dartmouth College \\ Curtis.L.Petersen.GR@Dartmouth. \\ edu \\ George G. Boateng \\ Department of Computer Science \\ Dartmouth College
}

\author{
Emily V. Wechsler \\ Thayer School of Engineering \\ Dartmouth College
}

\author{
Ryan J. Halter \\ Thayer School of Engineering \\ Dartmouth College
}

\author{
Patrick O. Proctor \\ Department of Computer Science \\ Dartmouth College
}

\author{
David F. Kotz \\ Department of Computer Science \\ Dartmouth College
}

\author{
Summer B. Cook \\ University of New Hampshire
}

\author{
John A. Batsis \\ The Dartmouth Institute \\ Dartmouth College \\ Darmtouth-Hitchcock Medical Center
}

\begin{abstract}
Sarcopenia is defined as an age-related loss of muscle mass and strength which impairs physical function leading to disability and frailty. Resistance exercises are effective treatments for sarcopenia and are critical in mitigating weight-loss induced sarcopenia in older adults attempting to lose weight. Yet, adherence to homebased regimens, which is a cornerstone to lifestyle therapies, is poor and cannot be ascertained by clinicians as no objective methods exist to determine patient compliance outside of a supervised setting. Our group developed a Bluetooth connected resistance band that tests the ability to detect exercise repetitions. We recruited 6 patients aged 65 years and older and recorded 4 specific, physical therapist-led exercises. Three blinded reviewers examined the findings and we also applied a peak finding algorithm to the data There were 16.6 repetitions per exercise across reviewers, with an intraclass correlation of 0.912 ( $95 \% \mathrm{CI}: 0.853-0.953, \mathrm{p}<0.001)$ between reviewers and the algorithm. Using this novel resistance band, we feasibly detected repetition of exercises in older adults.
\end{abstract}

\section{KEYWORDS}

Mobile Health, mHealth, Sarcopenia, Physical Therapy, Remote Monitoring, Digital Biomarker

\section{ACM Reference Format:}

Curtis L. Petersen, Emily V. Wechsler, Ryan J. Halter, George G. Boateng, Patrick O. Proctor, David F. Kotz, Summer B. Cook, and John A. Batsis. 2018. Detection and Monitoring of Repetitions Using an mHealth-Enabled Resistance Band. In ACM/IEEE International Conference on Connected Health:

Permission to make digital or hard copies of all or part of this work for personal or classroom use is granted without fee provided that copies are not made or distributed for profit or commercial advantage and that copies bear this notice and the full citation on the first page. Copyrights for components of this work owned by others than ACM must be honored. Abstracting with credit is permitted. To copy otherwise, or republish, to post on servers or to redistribute to lists, requires prior specific permission and/or a fee. Request permissions from permissions@acm.org.

CHASE '18, September 26-28, 2018, Washington, DC, USA

(C) 2018 Association for Computing Machinery.

ACM ISBN 978-1-4503-5958-0/18/09 . \$ \$15.00

https://doi.org/10.1145/3278576.3278586
Applications, Systems and Engineering Technologies (CHASE '18), September 26-28, 2018, Washington, DC, USA. ACM, New York, NY, USA, 3 pages. https://doi.org/10.1145/3278576.3278586

\section{INTRODUCTION}

Sarcopenia is the age-related loss of skeletal muscle mass and strength. [7] Mitigating its development is critically important in older adults to prevent functional limitations, disability and frailty, all of which increase the risk of nursing home placement and death. $[2,4,9]$ Exercise regimens are safe and effective treatment of sarcopenia, even in high-risk functionally impaired individuals and can lead to improvements in physical function. [6] Performing such exercises during significant weight loss efforts can prevent the loss of muscle mass and enhance physical performance. [1] The use of resistance bands is an integral part of any physical therapy program and can be used both in research and home-based settings.

While such evidence-based treatments are standard of care, as many as $40 \%$ of patients fail to adhere to treatment recommendations, including such exercise regimens. [3, 5] It is critical for clinical teams to know participants compliance and the number of recommended repetitions in such programs - the absence of which impacts treatment regimens for sarcopenia prevention. [3] Mobile health (mHealth) technologies that can communicate wirelessly to cloud-based computing systems are a potential solution for the evaluation and feedback to the treating provider. Other than directly observed, home-based physical therapy, we are unaware of methods that are able ascertain adherence to resistance exercises. The purpose of this study was to evaluate the feasibility of tracking specific exercises using a Bluetooth connected resistance band that could potentially impact adherence (completing the exercise) and quality (duration and frequency) of exercise-based interventions. As our interests lie in weight-loss induced sarcopenia, we purposely evaluated our device in individuals participating in an ongoing weight-loss study. 


\section{MATERIALS AND METHODS}

We conducted a feasibility evaluation in 6 older adults recruited through an existing weight-loss study (NCT03041831) whose purpose to lose weight and improve muscle strength. Maximal handgrip strength, a measure of upper extremity strength, was obtained using a JAMAR dynamometer.

The Bluetooth connected resistance band has been previously described. [8] Briefly, we used a commercially available Green TheraBand rated for $4.6 \mathrm{lbs}$ of resistance at $100 \%$ elongation. This tubing was connected to a commercial pressure sensor (Tekscan FlexiForce A201 sensor) with a detection range 0 -25lbs. Force was applied between the resistance tube?s end and the handle when the device was in use. Relative force was collected at a rate of 10 readings per second and sent to a smartphone via Low Energy Bluetooth to the Lightblue iPhone application. Participants performed four different exercises selected to promote muscle strengthening: elbow flexion; shoulder lift; seated rows; and triceps extension. Each exercise was carried out in succession with time for rest.

Data was examined with a peak detection algorithm that determined number of repetitions - defined as a peak of the measured relative force exerted on the connected exercise band. The algorithm consisted of a local regression (LOESS) smoothing with a second-degree polynomial term, calculating the change in slope between each reading, removing any peaks that did not exceed an minimum value. The total number of repetitions was defined as the sum of the number of peaks for that exercise. Noise was assessed as the ratio of the LOESS smoothed to recorded value; signal to noise ratio (SNR). For each exercise, we assessed the mean SNR.

Three independent individuals, blinded to the participant and exercise, examined the data, identifying the number of repetitions. We evaluated the reliability of the algorithm by calculating intraclass correlation coefficient between the human and algorithmdetermined repetition counts.

\section{RESULTS}

Mean age was $74 \pm 3.7$ years $(100 \%$ white females) with a mean body mass index of $35.7 \pm 5.19 \mathrm{kgMean}$ baseline grip strength was $22.2 \pm 5.8 \mathrm{~kg}$. All six subjects were able to use the Bluetooth connected resistance band after a demonstration of its use by an instructor. All were able to complete all 4 exercises and all 24 exercises yielded data that could be visually inspected and processed through an algorithm. Across all participants, the mean number of repetitions between all raters (3 blinded and 1 algorithm) was 22.3, 22.5, 13.3, and 9.8 (mean: 16.76, standard deviation 1.71 (range: 0.0-10.1)) for exercises $1,2,3$, and 4, respectively. The intraclass correlation between the blinded readers was 0.899 (95\%CI:0.824-0.947;p<0.001) and between blinded readers and the algorithm was 0.912 (95\%CI: 0.853-0.953; $\mathrm{p}<0.001)$. The mean difference between the maximal and minimal peak and the standard deviations for each of the four exercises was $66.71 \pm 42.19,34.00 \pm 30.74,18.00 \pm 10.75$ and $23.71 \pm 20.37$. Table 1 represents the algorithm derived characteristics.

\section{DISCUSSION}

The intent of our device was to provide an emerging mHealth strategy that could potentially be deployed within weight-loss treatment programs in older adults to prevent sarcopenia. Our system lays the
Table 1: Characteristics of Repetitions (Reps)

\begin{tabular}{ccccc}
\hline Exercise & $\begin{array}{c}\text { Repetitions } \\
\text { Mean (SD) }\end{array}$ & $\begin{array}{c}\text { Time Between } \\
\text { Mean (SD) }\end{array}$ & $\begin{array}{c}\text { SNR } \\
\text { Mean (SD) }\end{array}$ & $\begin{array}{c}\text { Active Time } \\
\text { Mean (SD) }\end{array}$ \\
\hline 1 & $22.57(6.21)$ & $29.73(4.82)$ & $1.29(0.27)$ & $1054.14(193.73))$ \\
2 & $22.86(11.81)$ & $29.79(7.08)$ & $1.20(0.18)$ & $942.00(128.93)$ \\
3 & $13.29(7.78)$ & $30.42(6.38)$ & $1.02(0.04)$ & $787.83(315.24)$ \\
4 & $7.75(6.69)$ & $80.17(45.91)$ & $1.06(0.09)$ & $1381.14(863.84)$ \\
\hline
\end{tabular}

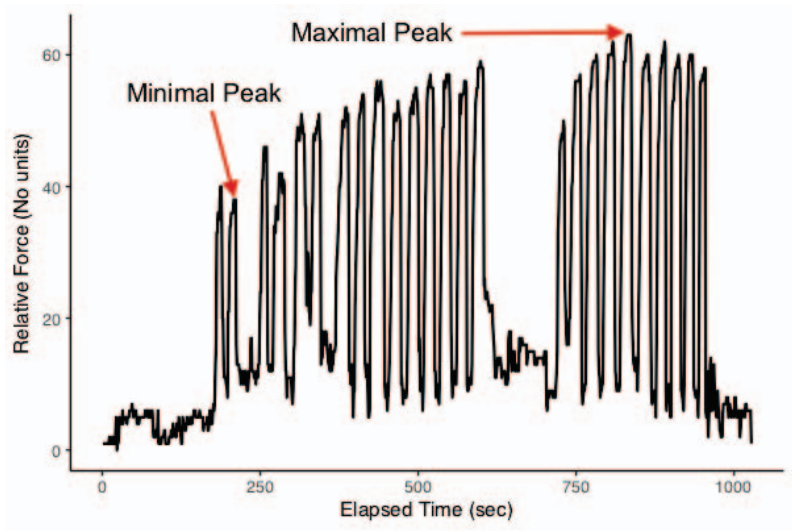

Figure 1: Example Recorded Force For Exercise

foundation to monitor an individual?s repetitions and compliance within a strength training regimen to treat sarcopenia. Participants completed the four exercises and high internal consistency was observed between the number of repetitions assessed by observers as compared to the peak-detection algorithm indicating that given this data the algorithm can monitor patient repetitions as well as a person.

The four exercises, as outlined by our physical therapy team, differed in difficulty evidenced by the number of mean repetitions the participants were able to conduct and the time between each of the repetitions. This was of particular significance for exercise 4 which targeted the triceps. The observed SNR suggests high variation from data point to data point due to the hardware, the participant?s movements, or both. This should be explored through further in-lab and user testing. Last, the standard deviation between our mini$\mathrm{mal} /$ maximal peaks within each exercise differed suggesting the dynamics of each exercise may need to be accounted for in future testing and could be used for prediction.

Our feasibility study holds considerable promise. Future study should evaluate the ability of the device to ascertain change of use over time. Subsequent modifications of this Bluetooth resistance band should account for the inter-person and inter-exercise variation which may differ through adjustment of both hardware and algorithm assessment. Importantly, the usability of this device in a home-based setting through a cloud-based infrastructure providing feedback to patients and providers are important next steps in the deployment of this device in clinical trials.

\section{CONCLUSION}

A simple Bluetooth connected resistance band can feasibly be a useful tool that will allow measurement of the number of repetitions 
per exercise which ultimately can be used to manage home-exercise regiments for sarcopenia with high consistency.

\section{ACKNOWLEDGMENTS}

The authors would like to thank all the patient participants and individuals who worked on the connected band device. Research was supported in part by the Burroughs Wellcome Fund, the National Institute On Aging of the National Institutes of Health K23AG051681, The Dartmouth Clinical and Translational Science Institute UL1TR001086 from the National Center for Advancing Translational Sciences (NCATS), the Health Promotion and Disease Prevention Research Center (Cooperative Agreement Number U48DP005018) from the Centers for Disease Control and Prevention, and the National Science Foundation (CNS-1314281, CNS-1314342, CNS-1619970, and CNS-1619950)

\section{REFERENCES}

[1] John A Batsis and Alexandra B Zagaria. 2017. Addressing Obesity in Aging Patients. Medical Clinics of North America (2017).

[2] A. B. Dufour, M. T. Hannan, J. M. Murabito, D. P. Kiel, and R. R. McLean. 2013 Sarcopenia definitions considering body size and fat mass are associated with mobility limitations: the Framingham Study. F Gerontol A Biol Sci Med Sci 68, 2 (2013), 168-74. https://doi.org/10.1093/gerona/gls109

[3] J. Hardage, C. Peel, D. Morris, C. Graham, C. Brown, H. R. Foushee, and J. Braswell 2007. Adherence to Exercise Scale for Older Patients (AESOP): a measure for predicting exercise adherence in older adults after discharge from home health physical therapy. I Geriatr Phys Ther 30, 2 (2007), 69-78.

[4] A. P. Marsh, W. J. Rejeski, M. A. Espeland, M. E. Miller, T. S. Church, R. A. Fielding T. M. Gill, J. M. Guralnik, A. B. Newman, and M. Pahor. 2011. Muscle strength and BMI as predictors of major mobility disability in the Lifestyle Interventions and Independence for Elders pilot (LIFE-P). F Gerontol A Biol Sci Med Sci 66, 12 (2011), 1376-83. https://doi.org/10.1093/gerona/glr158

[5] Leslie R. Martin, Summer L. Williams, Kelly B. Haskard, and M. Robin DiMatteo. 2005. The challenge of patient adherence. Therapeutics and Clinical Risk Management 1, 3 (2005), 189-199.

[6] M. Pahor, J. M. Guralnik, W. T. Ambrosius, and et al. 2014. Effect of structured physical activity on prevention of major mobility disability in older adults: The life study randomized clinical trial. FAMA 311, 23 (2014), 2387-2396. https: //doi.org/10.1001/jama.2014.5616

[7] Jeremy D. Walston. 2012. Sarcopenia in older adults. Current opinion in rheumatology 24, 6 (2012), 623-627. https://doi.org/10.1097/BOR.0b013e328358d59b

[8] Emily Wechsler. 2017. Development of a ÃćÂĂÂŸSmartÃćÂĂÂŹ Thera-Band to Asses Strength. (2017).

[9] Q. L. Xue, J. D. Walston, L. P. Fried, and B. A. Beamer. 2011. Prediction of risk of falling, physical disability, and frailty by rate of decline in grip strength: the women's health and aging study. Arch Intern Med 171, 12 (2011), 1119-21. https: //doi.org/10.1001/archinternmed.2011.252 